Tapia-Ruelas, C.S. (2021). Los buenos profesores universitarios, en la voz de las familias. Un insumo para el diseño de programas de formación docente con pertinencia social. Revista Electrónica Interuniversitaria de Formación del Profesorado, 24(3), 167-183.

DOI: https://doi.org/10.6018/reifop.469921

\title{
Los buenos profesores universitarios, en la voz de las familias. Un insumo para el diseño de programas de formación docente con pertinencia social
}

\author{
Claudia Selene Tapia Ruelas \\ Instituto Tecnológico de Sonora
}

\section{Resumen}

El objetivo de esta investigación fue describir las percepciones de padres y madres de familia acerca de las características de la enseñanza de los buenos profesores universitarios, consideradas como un insumo para el diseño de programas de formación docente con pertinencia social. Estudio cualitativo con indagación naturalista. Los participantes estuvieron integrados por 93 padres y madres de familia con hijos universitarios en México. Se empleó una guía de tópicos y una entrevista semiestructurada. Emergieron temáticas, de mayor a menor densidad fueron: a) crea clima agradable de confianza, b) explica claramente, c) preparados en su materia y la didáctica, d) enseña de forma entusiasta y práctica y e) apoya a los estudiantes en su aprendizaje. Las categorías en las que se agruparon las temáticas son: a) condiciones previas a la enseñanza y b) acciones durante la enseñanza. Los resultados de comparar las percepciones de los padres con otros estudios valida su voz y abre posibilidades de participación en los procesos de redefinición de políticas y contenidos en los procesos de formación docente.

\section{Palabras clave}

Palabras clave: Percepciones de las familias; Formación del Profesorado; Participación familiar; Docencia universitaria.

\section{Contacto:}

Claudia Selene Tapia Ruelas ctapia@itson.edu.mx, Departamento de Educación, Instituto Tecnológico de Sonora, calle 5 de Febrero 816 Centro, Ciudad Obregón, Sonora, México.

Investigación realizada con financiamiento del Programa de Fortalecimiento y Apoyo a la Investigación (PROFAPI, 2020), México. 


\title{
Good university professors, in the voice of families. An input for the design of teacher training programs with social relevance
}

\begin{abstract}
The objective of this research was to describe the perceptions of the families about the teaching characteristics of good university professors, considered as an input for the design of teacher training programs with social relevance. Qualitative study with naturalistic inquiry. The participants were made up of 93 parents with university children in Mexico. A topic guide and a semi-structured interview were used. The themes emerged, from highest to lowest density, were: a) creates a pleasant climate of trust, b) explains clearly, c) prepared in their subject and didactics, d) teaches enthusiastically and practically, and e) supports students in their learning. The categories in which the topics were grouped are: a) pre-teaching conditions and b) actions during teaching. The results of comparing the perceptions of parents with other studies validate their voice and open possibilities for participation in the processes of redefining policies and content in teacher training processes.
\end{abstract}

\section{Key words}

Keywords: Perceptions of families; Teacher training; Family participation; University teaching.

\section{Introducción}

La familia es considerada como un factor clave en los sistemas escolares. La Organización de las Naciones Unidades para la Educación la Ciencia y la Cultura, UNESCO (2018) en su agenda educativa 2030, recomienda a los gobiernos "redefinir las políticas, estrategias y lineamientos para la formación, el desempeño y la evaluación de los docentes" (p. 100). Para lograr este objetivo de una redefinición, se indica convocar a diversos actores educativos para maximizar la pertinencia social. Contar con información de lo que tienen para expresar la familia sobre la enseñanza universitaria, resulta útil ante este objetivo.

Desde hace décadas se ha resaltado la importancia del vínculo escuela-sociedad para mejorar la enseñanza en educación superior (UNESCO, 1998). Sobre ello, hay concordancia científica respecto al impacto que tiene el vínculo escuela-familia (Colás \& Contreras, 2013; Flórez, et al., 2017; Laboratorio Latinoamericano de Evaluación de la Calidad de la Educación [LLECE],1997; Madrid, et al., 2019; Rodríguez, et al., 2008; UNESCO, 2010). Pero también aún son escasas las referencias normativas que orienten el significado de operar las alianzas educativas (Gigli, et al., 2019). Hay quienes piensan que aún no se tiene un modelo de vinculación universal (Galián, et al., 2018). Sin embargo, hay posturas más abiertas, al señalar que la escuela-familia son ambas protagonistas en su propia construcción de roles y formas de relacionarse (Madrid, R., et al., 2019).

Este estudio se enfoca en indagar lo que los padres y madres de familia con hijos universitarios mexicanos, perciben como buen profesor universitario. Los resultados serán útiles a quienes toman decisiones en las instituciones de educación superior sobre diversos procesos, como la formación del profesorado y puede ser un insumo para los programas relacionados con ello. También se está de acuerdo con López (2011) al decir que las 
investigaciones de este tipo, aportan el sentido epistemológico y explicativo de cómo puede ser la práctica de los buenos profesores.

Existen estudios que concluyen que un buen profesor hace una gran diferencia en el aprendizaje de sus estudiantes, por otro lado, un mal profesor, puede generar daños irreversibles (Ravela, 2009). Específicamente, se considera la voz de los padres y madres de familia con hijos inscritos en el primer año universitario. Período que se ha reportado la mayor incidencia de abandono escolar.

Los porcentajes de abandono varían dependiendo del contexto, y estos van del $20 \%$ al $50 \%$ (Comisión Económica para América Latina y el Caribe [CEPAL], 2003; Parrino, 2010; Silva, 2011; Vries, et al., 2011). La familia sería más efectiva en la medida que sus esfuerzos educativos son pertinentes a las nuevas necesidades de sus hijos universitarios, donde se incluye el nexo escuela-y hogar, que falsamente parece que se rompe al ingresar a la educación superior (Solernou, 2013).

Este estudio es abordado desde una perspectiva interdisciplinar, entendida interdisciplina como el estudio de la base de los puntos de vista de las disciplinas pertinentes que pretenden lograr la integración real del conocimiento para una comprensión de fenómenos complejos (Newell, 2001).

Se consideraron las aportaciones de la teoría de la mejora del desempeño humano, la Educación, la teoría curricular y la línea de investigación de los buenos profesores, así como la teoría de la micropolítica. En la figura 1 se aprecian de manera general, las aportaciones y el tema de concurrencia.

\section{Figura 1.}

Perspectivas interdisciplinares para abordar el estudio: Los buenos profesores universitarios, en la voz de las familias. Un insumo social para diseño de programas de formación docente.

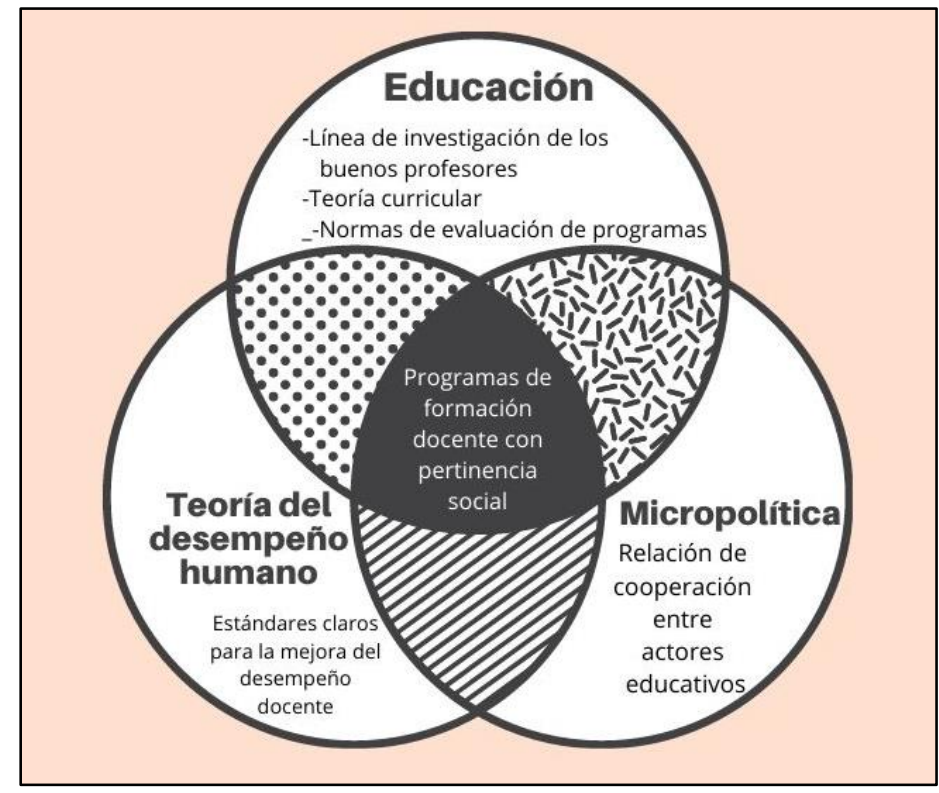

Fuente: elaboración propia

La teoría del desempeño humano, específicamente en el modelo de la Tecnología del Desempeño Humano o Human Performance Technology (HPT) indica que un factor clave son los estándares claros (Bernardez, 2009). Por su parte la teoría de micropolítica, en la 
premisa de Ball se señala que las escuelas concebidas como organizaciones, no se deberían estudiar como independientes del entorno (Bardisa, 1997). Es por ello que resalta la importancia de contar con la perspectiva de la familia en la construcción de estándares docentes.

Desde la teoría de la Educación, los programas educativos, deberían estar diseñados sobre la base de los fundamentos curriculares (Casarini, 2001). Al considerar a la familia en los programas de formación docente, se atendería un aspecto técnico de calidad que cualquier programa educativo debería cumplir: los fundamentos curriculares sociológicos. En la reforma educativa de la educación básica que se realizó en México en 2008, sí se consultó a padres y madres de familia en la fase de legitimación y validación de estándares, indagando si eran claros y pertinentes (Centro de Estudios Educativos, et al., 2013).

Otro aspecto técnico de calidad para el Joint Committee on Standards for Educational Evaluation (Comité Conjunto sobre estándares de Evaluación Educativa, 2008), es que todo programa educativo debe tomar en cuenta a sus audiencias, es decir, a las personas interesadas o afectadas por el mismo, cumpliendo así la norma de utilidad. La familia se integra a la denominación, de audiencia.

Continuando con el campo de la Educación, se tienen como marco de referencia la línea de investigación de la enseñanza de los buenos profesores. Contribuyó la definición del buen profesor de Bain (2007) como quienes, con sus acciones ayudan, también estimulan a los estudiantes a aprender, de tal manera que éstos consigan una diferencia muy positiva, sustancial y que es sostenida, tanto en la forma de pensar, actuar o la manera en que sienten, todo ello sin efectuarles algún tipo de daño apreciable. Otra propuesta relacionada con la enseñanza eficaz de los buenos profesores, es la de Martínez y Murillo (2016) quienes construyeron un modelo Iberoamericano, basado en evidencia empírica y puede constituir un referente para educación superior, aun cuando haya surgido en niveles educativos básicos. En el modelo se agrupan varios tópicos que resultaron asociados al aprendizaje de los estudiantes, en tres grandes categorías, estas son: a) Condiciones, como aspectos a considerar, previos a la enseñanza, b) Acciones, con tópicos como la metodología empleada por el docente durante la enseñanza y el clima del aula que genera y c) Resultados, estos se relacionan con el desarrollo cognitivo y socio-afectivo de los estudiantes y la implicación familiar.

En referencia a los estudios sobre el tema de los buenos profesores, se observa que, en cuanto a cantidad, abundan más estudios sobre las características del buen profesor relacionados con la perspectiva de los estudiantes y profesores, que con la de los padres y madres de familia. Se ha encontrado que los buenos profesores universitarios tienen dominio de su materia (Belando y Távarez 2017; Merellano, et al., 2016). Al igual Bain (2007) encontró en sus estudios con numerosas universidades que los buenos profesores conocen su materia extremadamente bien.

Así mismo, existen estudios que coinciden que los buenos profesores durante sus clases realizan explicaciones claras (Siqueira, 2015; Sgreccia, et al., 2016; Valdés y Collazo, 2013). A ello se agregan los hallazgos de Tapia, et al., (2017) que, al momento de explicar, el buen profesor es claro y presenta ejemplos reales de la vida cotidiana. Uno de los aspectos que también resaltan en los estudios es la idea de dinamismo, un buen profesor universitario desarrolla las clases de manera activa, con actividades prácticas y favorecen la aplicación de lo aprendido en la vida (Bain, 2007; Belando y Távarez, 2017; Tapia, et al., 2017).

En resumen, considerar la voz de los padres y madres de familia en cuanto al significado que le atribuyen al buen profesor universitario, puede constituir un insumo social que contribuye 
al contenido y al cumplimiento de criterios técnicos para los procesos de formación docente en educación superior.

\section{Metodología}

\section{Participantes}

Fueron 93 participantes, integrados por padres y madres de familia con hijos estudiando en la segunda universidad pública con mayor cantidad de estudiantes del Estado de Sonora, México, la cual cuenta con cuatro campus en varias ciudades del estado (Ciudad Obregón, Guaymas, Empalme, Navojoa). La selección de los padres y madres de familia se realizó a través de un muestreo por oportunidad, de acuerdo a quienes voluntariamente aceptaron y dieron su consentimiento.

De estos 93 padres y madres de familia, 68 respondieron una guía de tópicos. El 68\%(46) eran del género femenino y el otro $32 \%(22)$ del masculino. Sus edades se encontraban entre los 37 a 62 años de edad y en su mayoría tenían entre 40 y 50 años. De ellos, el 66\%(45) trabaja y el $34 \%(23)$ no trabaja. Respecto al nivel máximo de estudios de los 68 participantes el $6 \%(4)$ terminó la primaria, el 47\%(32) la secundaria, el 29\%(20) la preparatoria, el 15\%(10) la universidad y el 3\% (2) la maestría.

$Y$ el resto de padres y madres de familia que eran 25 , participó en una entrevista semiestructurada. De ellos el $72 \%$ (18) eran del género femenino y el otro $28 \%$ (7) era del masculino y se encontraban entre los 39 a 65 años de edad y en su mayoría tenían entre 40 y 50 años. De ellos, el 89\% (22) trabajan. Respecto al nivel máximo de estudios el 6\%(1) concluyó solo la primaria, el $28 \%$ (8) la secundaria, el $11 \%$ (3) preparatoria, un $44 \%$ (11) tienen una licenciatura y el $11 \%$ (2) maestría.

\section{Diseño}

Enfoque cualitativo naturalista. Este paradigma contribuye a la comprensión e interpretación de las realidades cotidianas (Fraile \& Vizcarra, 2009; Guba, 1989). Se concreta en un estudio de caso. En este paradigma cualitativo naturalista, los fenómenos están íntimamente vinculados a los momentos y los contextos en los que se asienta (Guba, 1989). El paradigma naturalista parte de la premisa que la teoría nace de los datos en sí mismos (Glaser y Strauss, 1967; citado por Guba, 1989).

\section{Instrumentos}

Se emplearon dos: 1) una guía de tópicos que respondieron los padres y madres de familia por medio de la técnica de la composición escrita. La composición es una técnica individual, consiste que el informante, a partir de una pregunta o tópico que se le entrega por escrito, realiza una composición escrita sobre el objeto de investigación, posteriormente se analiza el discurso desde sus experiencias y percepciones (Castillo y Oliveros, 2011). Y como segundo instrumento 2) una entrevista semiestructurada para responder de manera verbal, audio grabada. Ambos materiales contenían dos tópicos que se respondieron de forma abierta (ver tabla 1).

Tabla 1.

Especificaciones de categorías del estudio para la entrevista y guía de tópicos empleada, acerca de las características de la enseñanza de los buenos profesores universitarios.

\begin{tabular}{llll}
\hline Macro categoría & Definición conceptual & Micro categorías \\
\hline $\begin{array}{l}\text { Características de la } \\
\text { enseñanza de los buenos }\end{array}$ & $\begin{array}{l}\text { Características personales y a) } \\
\text { acciones del profesor }\end{array}$ & $\begin{array}{l}\text { Características de las clases de los } \\
\text { buenos profesores universitarios }\end{array}$ \\
profesores universitarios. & universitario que ayudan y & \\
\hline
\end{tabular}


estimulan a los estudiantes a

aprender. b) Características del buen profesor universitario

Fuente: elaboración propia.

\section{Procedimiento}

La inmersión al campo inició al solicitar a las autoridades del Departamento de Educación de la universidad las autorizaciones correspondientes y a la Comisión de Ética institucional.

En la primera fase de recolección de datos realizada en el año 2019 se utilizó una guía de tópicos aplicada por la investigadora de este estudio. La decisión muestral fue indagar con padres y madres de familia del primer año universitario, se aplicó a 68 participantes con hijos inscritos en el primer año que aceptaron y dieron su consentimiento informado. La aplicación se llevó a cabo durante reunión presencial en las instalaciones universitarias. Se transcribieron y analizaron las respuestas emitidas, de manera inductiva de acuerdo a las orientaciones de la Teoría Fundamentada (Glaser \& Strauss, 1967), validando que las interpretaciones se realicen en una constante comparación entre datos, reduciendo el riesgo de distorsión del significado (Strauss \& Corbin, 2002).

Se empleó el software MAXQDA2020 apegándose a un proceso de identificación de temas de forma inductiva simple, sin un patrón previo. En un esfuerzo por relacionar las temáticas que emergieron de los datos de este estudio, se recurrió a un modelo existente relacionado con la enseñanza eficaz (Martínez y Murillo, 2016) basados en estudios empíricos con estudiantes, al no contar en Latinoamérica uno relacionado con la familia, al menos en los últimos cinco años que se delimitó la búsqueda en bases de datos académicas. Se recurrió también al uso de memos durante todo el proceso.

Se decidió una segunda fase, en el año 2020 para lograr el objetivo del estudio, en apego al proceso para la saturación teórica (Ardila y Rueda, 2013). Se empleó una entrevista semiestructura con las mismas preguntas de la fase anterior a 25 padres y madres de familia que aceptaron participar, a través de una invitación enviada con sus hijos universitarios. Este proceso implicó más tiempo para conseguir la aceptación de los papás, se sumaron, a un total de 93 participantes, logrando la saturación. Debido a la pandemia por coronavirus COVID-19, la entrevista con los participantes fue realizada a distancia, con dispositivos electrónicos.

Las consideraciones éticas tuvieron que ver con el criterio de credibilidad de la investigación naturalista, mediante la inclusión en el estudio de citas literales de los padres y madres de familia representativas de la categoría definida y su densidad -frecuencia y concurrencia-. Por otro lado, el análisis se realizó entre dos investigadores, ambos con formación en Ciencias de la Educación, logrando la consistencia lógica interna (Salgado, 2007) o lo que Guba (1989) señala como réplica paso a paso. Para la confidencialidad, se emplearon homoclaves, para el anonimato de los participantes.

Se optó por un proceso muy específico de obtención de validez externa de las categorías identificadas con los padres y madres de familia, para ello se realizó una búsqueda con operadores booleanos en bases de datos académicas y Google académico de estudios relacionados con el tema de los buenos profesores, realizados exclusivamente en diez de las primeras mejores universidades ubicadas en uno de los principales rankings académicos latinoamericano (Folch, et al., 2015) el QS Latin América Rankings (2019). Ver tabla 2. 
Tabla 2.

Universidades posicionadas en los diez primeros lugares del ranking académico latinoamericano QS Latin América Rankings, 2019.

\begin{tabular}{lll}
\hline Universidades & País & $\begin{array}{c}\text { QS Latin } \\
\text { Rankings }\end{array}$ \\
\hline Pontificia Universidad Católica de Chile & Chile & 1 \\
Universidade de Sao Paulo & Brasil & 2 \\
Universidade Estadual de Campinas & Brasil & 3 \\
Universidad Nacional Autónoma de México & México & 4 \\
Universidad de los Andes & Colombia & 5 \\
Instituto Tecnológico y Estudios Superiores de Monterrey & México & 6 \\
Universidad de Chile & Chile & 7 \\
Universidad de Buenos Aires & Argentina & 8 \\
Universidade Federal do Rio de Janeiro (Brasil). & Brasil & 9 \\
Universidad Nacional de Colombia & Colombia & 10 \\
\hline
\end{tabular}

Fuente: elaboración propia

Se encontraron los estudios y se procedió al análisis inductivo del contenido (Strauss \& Corbin, 2002) para la identificación de las categorías de estos estudios y posteriormente compararlas con las obtenidas con las respuestas de los padres y madres de familia.

\section{Resultados}

En la figura 2 se presentan las temáticas que emergieron de los datos para caracterizar la enseñanza del buen profesor universitario. Fueron construidas a partir de las respuestas literales/textuales de los 93 participantes, constituidos por padres y madres de familia con hijos universitarios. Se presentan como temáticas en este primer momento, para mostrar el apego a los datos. Las categorías en las que se agruparon posteriormente estas temáticas, fueron de acuerdo al modelo de enseñanza eficaz de Martínez y Murillo (2016), estas son: a) Condiciones previas a la enseñanza y b) Acciones del profesor durante la enseñanza, como se puede observar en la figura.

Es importante señalar que la tercer categoría del modelo de estos investigadores denominada Resultados de la enseñanza, se identificó parcialmente en este estudio en la temática: Vincula con los padres de familia, visualizada en la figura, la cual solo contó con cinco menciones. Esta categoría también tiene relación con los resultados en el desarrollo cognitivo y socio-afectivo de los estudiantes (Martínez y Murillo, 2016). Aspectos que en este estudio no fueron considerados por los participantes.

También se presentan citas literales/textuales retomadas de las respuestas de los padres y madres de familia y las temáticas en las que se agruparon, como evidencia del criterio de credibilidad de los estudios de tipo naturalista (ver tabla 3). Se emplearon las siguientes homoclaves, PG significa: participante grupo focal y PE: participante entrevistado, seguido del número asignado a cada uno. 
Figura 2.

Características de la enseñanza del buen profesor universitario. La voz de las familias.

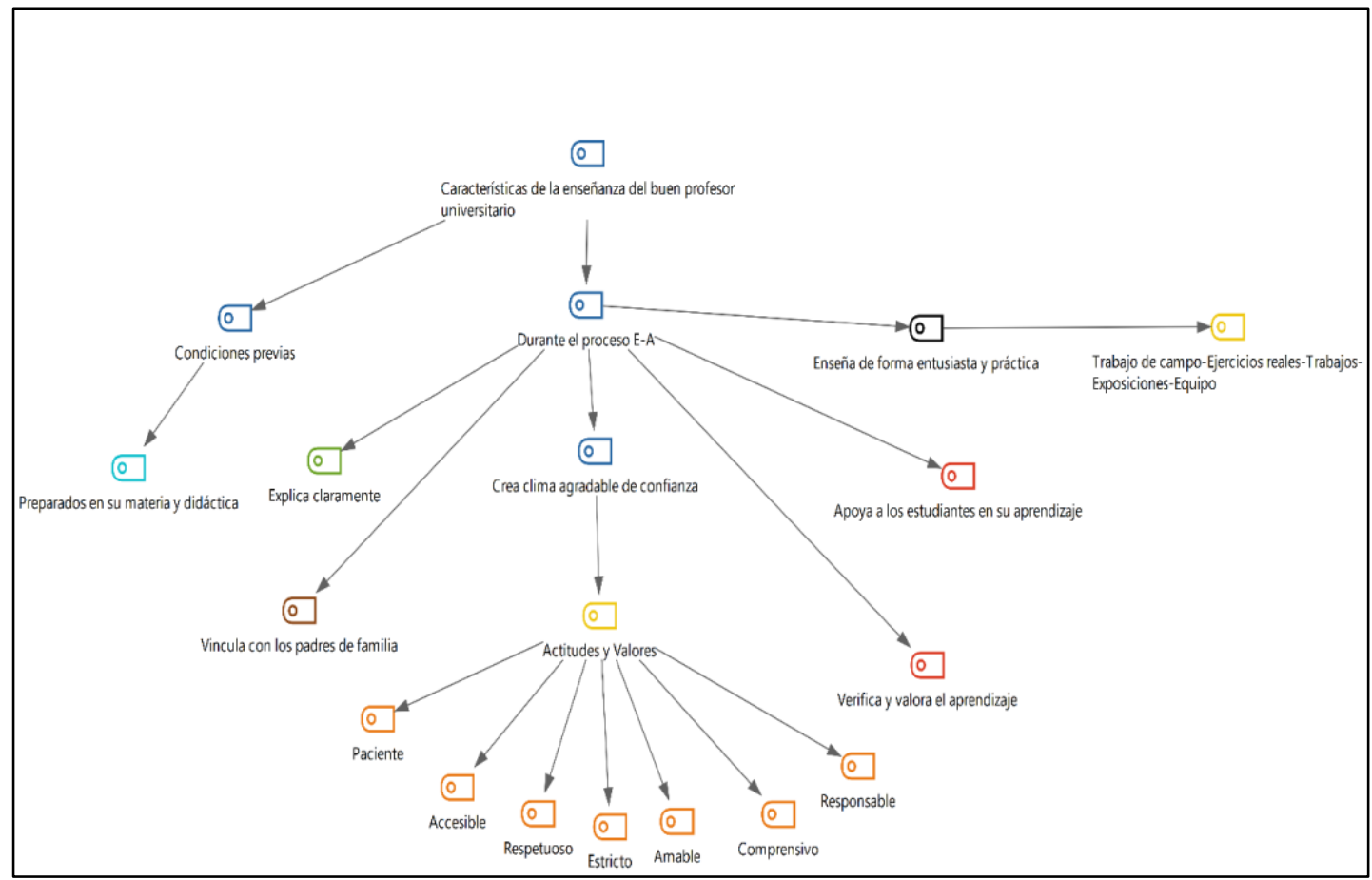

Fuente: elaboración propia

Se retoman las cinco temáticas que resultaron con mayor densidad por su frecuencia y concurrencias y en la columna final se especifica la categoría del modelo de enseñanza eficaz de Martínez y Murillo (2016) con la cual se relacionó cada temática identificada (ver tabla 3). Entre paréntesis se incluye la cantidad de veces que fueron referidas por los participantes y están ordenadas de mayor a menor densidad, estas son: a) crea clima agradable de confianza(135), b) explica claramente(39), c) preparados en su materia y la didáctica(36), d) enseña de forma entusiasta y práctica(23) y e) apoya a los estudiantes en su aprendizaje(13). Las temáticas denominadas verifica y valora el aprendizaje(5) y g) vincula con la familia(5) son las que tuvieron menor densidad. 
Tabla 3.

Citas literales de las respuestas retomadas de las entrevistas y guía de tópicos aplicadas a padres y madres de familia sobre las características de la enseñanza de los buenos profesores universitarios.

\begin{tabular}{|c|c|c|c|}
\hline $\begin{array}{l}\text { Citas literales extraídas de las } \\
\text { respuestas de los padres y madres } \\
\text { de familia }\end{array}$ & Aspectos & Temática & Categoría \\
\hline $\begin{array}{l}\text { PG33... "Dar confianza a los } \\
\text { estudiantes, tenerles paciencia, ya } \\
\text { que muchos de los jóvenes con } \\
\text { mucho esfuerzo han llegado hasta } \\
\text { este nivel" } \\
\text { PG24..."Buen ambiente del aula, } \\
\text { amable, hacerlos sentir confiados y } \\
\text { tranquilos" }\end{array}$ & $\begin{array}{l}\text { Paciente(39) } \\
\text { Respetuoso(31) } \\
\text { Accesible (21) } \\
\text { Amable(20) } \\
\text { Estricto(14) } \\
\text { Responsable(11) } \\
\text { Comprensivo(9) }\end{array}$ & $\begin{array}{l}\text { 1. Crea clima } \\
\text { agradable de } \\
\text { confianza en el } \\
\text { aula(135) }\end{array}$ & $\begin{array}{l}\text { Acciones } r \text { del } \\
\text { profesor durante } \\
\text { la enseñanza }\end{array}$ \\
\hline $\begin{array}{l}\text { PE2... "Son claros al explicar usando } \\
\text { ejemplos" } \\
\text { PG37... "Explica de forma fácil de } \\
\text { entender" }\end{array}$ & & $\begin{array}{l}\text { 2. Explica } \\
\text { claramente(39) }\end{array}$ & $\begin{array}{l}\text { Acciones del } \\
\text { profesor durante } \\
\text { la enseñanza }\end{array}$ \\
\hline $\begin{array}{l}\text { PE6..."Preparado en lo que va } \\
\text { impartir" } \\
\text { PE11..."Debe estar preparado no } \\
\text { solo en lo de su materia si no para } \\
\text { dar clases" }\end{array}$ & & $\begin{array}{l}\text { 3. Preparado en } \\
\text { su materia y la } \\
\text { didáctica(36) }\end{array}$ & $\begin{array}{l}\text { Condiciones } \\
\text { previas a la } \\
\text { enseñanza }\end{array}$ \\
\hline $\begin{array}{l}\text { PG8..." "Sus clases deben ser } \\
\text { amenas, con entusiasmo" } \\
\text { PG12..."Hace que realicen prácticas } \\
\text { reales y en empresas" } \\
\text { PG3... "Mucho trabajo y ejercicios } \\
\text { reales" }\end{array}$ & 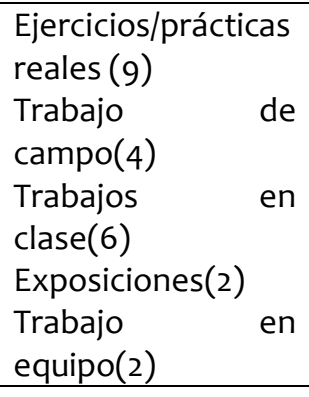 & $\begin{array}{ll}\text { 4. Enseña } & \text { de } \\
\text { forma } & \\
\text { entusiasta } & y \\
\text { práctica(23) } & \end{array}$ & $\begin{array}{lr}\text { Acciones } & \text { del } \\
\text { profesor durante } \\
\text { la enseñanza }\end{array}$ \\
\hline $\begin{array}{l}\text { PG58 "Que identifique a los alumnos } \\
\text { [ejemplo tímidos] y les de apoyo } \\
\text { incondicional en el aula" } \\
\text { PE18 "Sea sensible a la problemática } \\
\text { [de los estudiantes] y ayudarlos } \\
\text { cuando no entiendan" }\end{array}$ & & $\begin{array}{l}\text { 5. Apoya a los } \\
\text { estudiantes en } \\
\text { su } \\
\text { aprendizaje(13) }\end{array}$ & $\begin{array}{lr}\text { Acciones } & \text { del } \\
\text { profesor durante } \\
\text { la enseñanza }\end{array}$ \\
\hline
\end{tabular}

Como parte del proceso de validación de la voz de los padres y madres de familia, se hizo el comparativo con un referente externo y sobre ello se encontraron estudios similares de los buenos profesores, realizados en universidades ubicadas en cuatro países: Chile, México,

Colombia y Brasil. Se ha marcado con una cruz $(X)$ las coincidencias entre estos estudios, por considerarlo importante y como una forma de visualización resumida (ver tabla 4). 
Tabla 4.

Resumen de categorías derivadas de los estudios sobre el tema de los buenos profesores universitarios en las universidades ubicadas en los primeros 10 lugares del ranking latinoamericano QS Latin América Rankings, 2019.

\begin{tabular}{|c|c|c|c|c|c|c|c|c|c|c|}
\hline \multirow[t]{2}{*}{ Temáticas } & $\begin{array}{l}\text { Gallardo } \\
\& \text { Reyes } \\
\text { (2010) }\end{array}$ & $\begin{array}{l}\text { López } \\
\text { (2011) }\end{array}$ & $\begin{array}{l}\text { Guzmán } \\
\text { (2015) }\end{array}$ & $\begin{array}{l}\text { Guzmán } \\
\text { (2018) }\end{array}$ & $\begin{array}{l}\text { García y y } \\
\text { Medécigo } \\
\text { (2014) }\end{array}$ & $\begin{array}{l}\text { Hickman, } \\
\text { Alarcón, } \\
\text { Cepeda, } \\
\text { Cabrera y } \\
\text { Torres } \\
\text { (2016) }\end{array}$ & $\begin{array}{l}\text { Ramírez y } \\
\text { Montoya } \\
\text { (2018) }\end{array}$ & $\begin{array}{l}\text { Valerio y } \\
\text { Rodríguez } \\
(2017)\end{array}$ & $\begin{array}{l}\text { Galván y } \\
\text { Farías } \\
\text { (2018) }\end{array}$ & 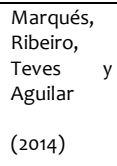 \\
\hline & $\begin{array}{l}\text { Chile } \\
\text { (1) }\end{array}$ & $\begin{array}{l}\text { México } \\
\text { (4) }\end{array}$ & $\begin{array}{l}\text { México } \\
\text { (4) }\end{array}$ & $\begin{array}{l}\text { México } \\
\text { (4) }\end{array}$ & $\begin{array}{l}\text { México } \\
\text { (4) }\end{array}$ & $\begin{array}{l}\text { México } \\
\text { (4) }\end{array}$ & $\begin{array}{l}\text { Colombia } \\
\text { (5) }\end{array}$ & $\begin{array}{l}\text { México } \\
\text { (6) }\end{array}$ & $\begin{array}{l}\text { México } \\
\text { (6) }\end{array}$ & $\begin{array}{l}\text { Brasil } \\
\text { (9) }\end{array}$ \\
\hline $\begin{array}{l}\text { Didáctica } \\
\text { práctica }\end{array}$ & & & $\mathrm{x}$ & $\mathrm{x}$ & $\mathrm{x}$ & $\mathrm{x}$ & $\mathrm{x}$ & $\mathrm{x}$ & $\mathrm{x}$ & $\mathrm{x}$ \\
\hline $\begin{array}{l}\text { Buena } \\
\text { relación con } \\
\text { los } \\
\text { estudiantes }\end{array}$ & $x$ & $\mathrm{x}$ & $\mathrm{x}$ & $\mathrm{x}$ & $x$ & $\mathrm{x}$ & & $\mathrm{x}$ & $\mathrm{x}$ & $x$ \\
\hline $\begin{array}{l}\text { Alto dominio } \\
\text { de su materia }\end{array}$ & & $\mathrm{x}$ & & $\mathrm{x}$ & $\mathrm{x}$ & $\mathrm{x}$ & & & $\mathrm{x}$ & $\mathrm{x}$ \\
\hline $\begin{array}{l}\text { Explicaciones } \\
\text { claras }\end{array}$ & & & $x$ & $\mathrm{x}$ & & & & $\mathrm{x}$ & & \\
\hline $\begin{array}{l}\text { Muestran } \\
\text { pasión y } \\
\text { entusiasmo }\end{array}$ & & $\mathrm{x}$ & & & $\mathrm{x}$ & & $\mathrm{x}$ & $\mathrm{x}$ & & $\mathrm{x}$ \\
\hline
\end{tabular}

Fuente: elaboración propia.

Se puede observar que las coincidencias tienen que ver con la relación con sus estudiantes, explicaciones claras, dominio de su materia, didáctica práctica y el entusiasmo. En síntesis, se observó que coinciden en cuatro de las cinco categorías de la tabla 3 y 4, lo cual representa un $80 \%$.

\section{Discusión y conclusiones}

El objetivo de este estudio era describir las percepciones de padres y madres de familia acerca de las características de la enseñanza de los buenos profesores universitarios. Se identificaron dos categorías en las cuales se logró agrupar las temáticas que se obtuvieron como resultado, de acuerdo al modelo Iberoamericano de enseñanza de Martínez y Murillo (2016).

\section{Condiciones previas a la enseñanza}

En este estudio, se identificó que los padres y madres de familia se refieren al dominio didáctico y disciplinar como condición previa a la enseñanza de los buenos profesores universitarios.

1. Preparado en su materia y la didáctica. Esta categoría, parece indicar que para los participantes es tan importante que dominen el tema, al igual que cómo enseñarlo a los estudiantes, quí lo mencionan, PE11... "Debe estar preparado no solo en lo de su materia si no para dar clases". Este aspecto ha sido reportado en otros estudios, como el de Bain (2007) y hallazgos similares en estudios acerca de la enseñanza eficaz, como el mostrar un dominio alto del contenido que enseña (Belando y Távarez 2017; Merellano, et al., 2016; Tapia, et al., 2017). 


\section{Acciones durante la enseñanza}

En esta categoría, se refieren a aquellas acciones didácticas que, al realizarlas el profesor, pueden lograr que aprendan sus hijos; y que en esas acciones se deben poner en práctica un conjunto de valores y actitudes específicos.

2. Crea clima agradable de confianza. Esta temática representa la de mayor densidad en el presente estudio, se logra apreciar un conjunto de actitudes y valores que los padres y madres de familia consideran que caracterizan a un buen profesor universitario (paciente, respetuoso, accesible, amable, estricto, responsable y comprensivo). Así los estudiantes tendrán la confianza de realizar preguntas, externar sus inquietudes y no percibirlos como impacientes o intolerantes. Se incluye el comportarse de forma Paciente que fue la subcategoría más mencionada. Como lo muestra este participante PG33... "Dar confianza a los estudiantes, tenerles paciencia, ya que muchos de los jóvenes con mucho esfuerzo han llegado hasta este nivel". Resultados que concuerdan con otros estudios en cuanto al clima de confianza en clase (Casillas, Cabezas y Pinto 2016; Hamer 2015) y resaltar la buena relación con los estudiantes como un aspecto de los buenos profesores (Guzmán, 2015; Gallardo \& Reyes, 2010; López, 2011).

Lo anterior significa para los profesores que imparten clases en el primer año de la universidad que se deberían desarrollar o consolidar saberes de tipo afectivo en su formación inicial o continua, y la sensibilidad de diferenciar las necesidades de los estudiantes, conforme avanzan en su trayectoria académica.

3. Explica claramente. Los participantes se refieren a explicar de manera clara, detallada, uso de elementos visuales y ejemplos del contexto. Sobre esta característica de la enseñanza del buen profesor, posiblemente los participantes la consideran como una forma de asegurar que sus hijos estén comprendiendo durante las clases, además esto, contribuye a asegurar una calificación aprobatoria, lo que significa que un trayecto académico regular, marca el éxito o el fracaso escolar (Zayas, et al., 2011). Este resultado Zabalza (2003) lo asociaría a la competencia comunicativa que debe poseer el profesor universitario.

4. Enseña de forma entusiasta y práctica. Los padres y madres de familia posiblemente tienen la certeza de que una habilidad es producto de la práctica y que si no se aplica lo aprendido los estudiantes no se apropiarán de las competencias que se espera en su carrera como lo hace notar este participante PG12 "Hace que realicen prácticas reales y en empresas". Lo anterior se asocia a un enfoque competencial y de aprendizaje activo. Asimismo, Murillo, Martínez-Garrido y Hernández-Castilla (2011) crearon un decálogo de la enseñanza eficaz y en uno de sus preceptos mencionan que el profesor debe desarrollar actividades variadas, participativas y activas.

5. Apoya a los estudiantes en su aprendizaje. Por los lazos afectivos con sus hijos, parece deseable que ellos reciban las ayudas pedagógicas durante el desarrollo de las clases como lo indica este participante PG57..." Ayuden [a los estudiantes] a conseguir sus metas". También se puede observar en el siguiente participante PE18 "Sea sensible a la problemática [de los estudiantes] y ayudarlos cuando no entiendan". Los mismos estudiantes han opinado, eso en otros estudios (Sayós, Pagés, Amador y Jorba, 2014) señalando que una de las características que debería tener un buen profesor es la disponibilidad y accesibilidad, consideran que el profesor debe atender las necesidades personales y de aprendizaje de los alumnos.

Como se puede apreciar resultó que los participantes tienen significados de lo que es la enseñanza de los buenos profesores y no están lejanos a lo que en estudios de otras universidades se ha encontrado, ni a los modelos de enseñanza que se han construido en 
Iberoamérica. Estos resultados parecen indicar que los padres y madres de familia están en sintonía con otros actores educativos importantes del campo educativo en Latinoamérica.

Llama la atención el peso que le han otorgado los participantes a aspectos relacionados con el modelo Iberoamericano de enseñanza eficaz, específicamente con la categoría: condiciones previas de la enseñanza, la cual tienen que ver en este estudio, con el dominio disciplinar y didáctico. Pero aún más peso se le dio, a la categoría acciones durante la enseñanza, enfatizando el dominio didáctico. Siendo lo más reiterado: el clima afectivo de clase. Un clima que el mismo profesor propicia con sus actitudes según los participantes. Sobresale el ser paciente, respetuoso y accesible como algo importante para los padres y madres de familia. Así mismo el explicar claramente. Se puede decir que independientemente de su nivel de estudios y edad, coincidieron los participantes en estos elementos.

Habría que indagar en futuros estudios el por qué no tuvo peso la categoría del modelo Iberoamericano de enseñanza eficaz Resultados de la enseñanza, al no identificarse como categoría saturada. Esta se refiere a resultados esperados en cuanto al desarrollo cognitivo y socio-afectivo de los estudiantes, exceptuando el vinculo con la familia, que sí se mencionó por los participantes como aspecto deseable en los buenos profesores, pero tuvo muy baja densidad.

Los hallazgos de este estudio son valiosos en los procesos de formación docente con pertinencia social y podrían contribuir al objetivo de la agenda educativa de la UNESCO (2018) de ampliar la convocatoria a los demás actores educativos, en la redefinición de políticas, estrategias y lineamientos para la formación de los docentes. Los padres y madres de familia son audiencias clave, interesadas o afectadas por el servicio educativo que reciben sus hijos, sobre todo por aquellos quienes inician la educación superior, debido al riesgo de abandono escolar, por lo que su perspectiva, puede enriquecer los procesos de todo proceso de formación del profesorado.

\section{Referencias}

Ardila, E. E., \& Rueda, J. F. (2013). La saturación teórica en la teoría fundamentada: su delimitación en el análisis de trayectorias de vida de víctimas del desplazamiento forzado en Colombia. Revista Colombiana de Sociología, 36(2), 93-114. https://revistas.unal.edu.co/index.php/recs/article/view/41641

Bain, K. (2007). Los que hacen los mejores profesores de universidad ( $2^{\mathrm{a}}$. Ed.). Barcelona: Universidad de Valencia. https://www.fceia.unr.edu.ar/geii/maestria/2014/DraSanjurjo/8mas/Ken\%20Bain,\%20L o\%20que\%2ohacen\%20los\%20mejores\%2oprofesores\%20de\%2ouniversidad.pdf

Bardisa, R., T. (1997). Teoría y práctica de la micropolítica en las organizaciones escolares. Revista Iberoamericana de Educación. Organización de Estados Iberoamericanos, OIT, (15). https://www.oei.es/historico/oeivirt/rie15a01.htm

Belando, M.R., \& Távarez, M. T. (2017). El perfil del docente en la educación universitaria de República Dominicana. Experiencia de una colaboración Erasmus Mundus entre la UCM y la UASD, Revista Internacional de Investigación e Innovación Educativa, 7, 164185.

https://www.researchgate.net/publication/311529774_El_perfil_del_docente_en_la_ educacion_universitaria_de_Republica_Dominicana_Experiencia_de_una_colaborac ion_Erasmus_Mundus_entre_la_UCM_y_la_UASD 
Bernardez, L.M. (2009). Desempeño Humano Manual de consultoría. USA: Editorial AuthorHouse.

Casarini, M. (2001). Teoría y diseño curricular. México: Trillas.

Casillas, S., Cabezas, M., \& Pinto, A. M. (2016). ¿Qué características psicológicas valoran los estudiantes universitarios de sus profesores?. RIDU. Revista Digital de Investigación en Docencia Universitaria, 10(2), 1-16. https://doi.org/10.19083/ridu.10.466

Castillo, E., y Oliveros, L. (2011). Competencias metodológicas desde la perspectiva cualitativa para la generación de conocimiento en comunicación y educación. En G. León, Estudios de la comunicación. Estrategias metodológicas y competencias profesionales en comunicación, 101-110. México: PEARSON, Universidad de Sonora.

Centro de Estudios Educativos, \& Servicios Integrales de Evaluación y Medición Educativa (SIEME), \& Heurística Educativa, S. C., (2013). Estándares para la educación básica. Experiencia de mejora continua en escuelas mexicanas del nivel básico, con base en estándares curriculares, de desempeño docente y gestión escolar. Revista Latinoamericana de Estudios Educativos (México), 43(3),23-72. https://www.redalyc.org/articulo.oa?id=27028898002

CEPAL (2003). Elevadas tasas de deserción escolar en América Latina. Panorama Social de América Latina 2001-2002. Santiago de Chile, Comisión Económica para América Latina y el Caribe. https://www.cepal.org/cgibin/getProd.asp?xml=/prensa/noticias/comunicados/0/11260/P11260.xml

Colás, B., P., \& Contreras, R., J., A. (2013). La Participación de las familias en los Centros de Educación Primaria. Revista de Investigación Educativa, 31(2),485499. https://doi.org/10.6018/rie.31.2.171031

Flórez, G., Villalobos, J., Londoño-Vásquez, D. (2017). El acompañamiento familiar en el proceso de formación escolar para la realidad colombiana: de la responsabilidad a la necesidad. Revista Psicoespacios, 11(18). https://doi.org/10.25057/21452776.888

Folch, M. T., Feixas, M., Bernabeu-Tamayo, M. B., \& Ruiz, J. M. (2015). La literatura científica sobre rankings Universitarios: una revisión sistemática. REDU. Revista de Docencia Universitaria, 13(3), https://polipapers.upv.es/index.php/REDU/article/view/5418/5398

33-54.

Fraile, A. \& Vizcarra, M., T. (2009). La investigacion naturalista e interpretativa desde la actividad fisica y el deporte. Revista de Psicodidáctica, 14(1) ,119132. https://ojs.ehu.eus/index.php/psicodidactica/article/view/254

Galián, N., B., García, S., M. P., \& Belmonte, A., M. L. (2018). Evaluación de la participación familiar en el proceso educativo de los discentes desde la percepción del profesorado. Revista Electrónica Interuniversitaria De Formación Del Profesorado, 21(3), 45-62. https://doi.org/10.6018/reifop.21.3.336971

Galván Salinas, J. O. y Farías Martínez, G. M. (2018). Características personales y práctica docente de profesores universitarios y su relación con la evaluación del desempeño. Revista Iberoamericana de Evaluación Educativa, 11(2), 9-33. https://doi.org/10.15366/riee2018.11.2.001 
Gallardo, G., \& Reyes, P. (2010). Relación profesor-alumno en la universidad: arista fundamental para el aprendizaje. Revista Calidad en la Educación, 32, 79-106. http://www.cned.cl/node/4478

Gigli, A., Demozzi, S., \& Pina, C., M. (2019). La alianza educativa escuela/familia y los grupos de chat de padres: una mirada a la situación italiana . Revista Electrónica Interuniversitaria de Formación Del Profesorado, 22(3), 15-30. https://doi.org/10.6018/reifop.389271

Glaser, B. \& Strauss, A. (1967). The discovery of grounded theory. Chicago: Aldine Press.

Guba, E., G. (1989). Criterios de credibilidad en la investigación naturalista. En: Gimeno, S., J. y Pérez, Á. (Eds). La enseñanza: su teoría y su práctica. (pp. 148-165). Madrid: Akal

Guzmán, J. J. C. (2015). Las buenas prácticas de enseñanza de los profesores de la Facultad de Ingeniería de la UNAM. (Tesis de maestría). UNAM. México. https://repositorio.unam.mx/contenidos/las-buenas-practicas-de-ensenanza-de-losprofesores-de-la-facultad-de-ingenieria-de-la-unam-

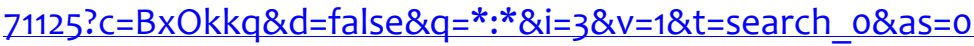

Guzmán, J. J. C. (2018). Las buenas prácticas de enseñanza de los profesores de Educación Superior. REICE. Revista Iberoamericana sobre Calidad, Eficacia y Cambio en Educación, 16(2), 133-149. https://doi.org/10.15366/reice2018.16.2.008

Hamer, A. (2015). La percepción del buen profesor en alumnos de nuevo ingreso a la enseñanza universitaria: el caso de ETEA (Córdoba). Revista Complutense de Educación, 26(2), 227-240. http://dx.doi.org/10.5209/rev_RCED.2015.v26.n2.41534

Hickman, H., Alarcón, M. E., Cepeda, M. L., Cabrera, R., \& Torres, X. K. (2016). Significado de un buen profesor y de evaluación docente por estudiantes y maestros universitarios. La técnica de redes semánticas. Sinéctica, Revista Electrónica de Educación, 47, 1-16. http://www.redalyc.org/pdf/998/99846346004.pdf

Joint Committee on Standards for Educational Evaluation (2008). Normas de evaluación para programas, proyectos y material educativo. México: Trillas.

Laboratorio Latinoamericano de Evaluación de la calidad de la educación, LLECE, de la Oficina Regional de Educación para América Latina y el Caribe, OREALC (1997). Marco Conceptual. http://unesdoc.unesco.org/images/0018/001836/183650s.pdf

López, A. Y. (2011). ¿Quién es un buen docente en la universidad? La perspectiva de los estudiantes de pedagogía del sistema de universidad abierta y a distancia en la UNAM. En H. Casanova (Presidencia), XI Congreso Nacional de Investigación Educativa. Congreso de la UNAM, México. http://www.comie.org.mx/congreso/memoriaelectronica/v11/docs/area_02/0685.pdf

Madrid, R., Saracostti, M., Reininger, T., \& Hernández, M. T. (2019). Responsabilización, obediencia y resistencia: perspectivas de docentes y padres sobre la colaboración familia-escuela. Revista Electrónica Interuniversitaria de Formación del Profesorado, 22(3), 1-13. https://doi.org/10.6018/reifop.389801 
Marqués, C., Ribeiro, M., Tevés, N., \& Aguilar, M. (2014). A Representação Social Do "Bom Professor" No Ensino Superior. Psicología \& Sociedade, 26(2), 356-365. http://www.scielo.br/pdf/psoc/v26n2/a12v26n2.pdf

Martínez-Garrido, C., \& Murillo, F. J. (2016). Investigación iberoamericana sobre enseñanza eficaz. Revista mexicana de investigación educativa, 21(69), 471-499. http://www.scielo.org.mx/scielo.php?script=sci_arttext\&pid=S140566662016000200471

Merellano, E., Almonacid, A., Moreno, A., \& Castro, C. (2016). Buenos docentes universitarios: ¿Qué dicen los estudiantes?. Revista Educ. Pesqui. 42(4). https://doi.org/10.1590/s15179702201612152689

Murillo, J., Martínez, C., \& Hernández, R. (2011). Decálogo para una enseñanza eficaz. Revista iberoamericana sobre Calidad, Eficacia y Cambio en educación, 1(9). http://www.rinace.net/reice/numeros/arts/volgnum1/art1.pdf

Newell, W. (2001). A Theory of Interdisciplinary Studies. ISSUES in integrative studies, 1-25.

Organización de las Naciones Unidades para la Educación la Ciencia y la Cultura, UNESCO, (1998). Declaración mundial sobre la educación superior en el siglo XXI: visión y acción. La educación superior en el siglo XXI: Visión y acción. http://www.iesalc.unesco.org/ess/index.php/ess3/issue/view/21

Organización de las Naciones Unidas para la Educación, la Ciencia y la Cultura, UNESCO (2010). Factores asociados al logro cognitivo de los estudiantes de América Latina y el Caribe. Laboratorio Latinoamericano de Evaluación de la Calidad de la Educación, LLECE. http://unesdoc.unesco.org/images/0018/001867/186769S.pdf

Organización de las Naciones Unidades para la Educación la Ciencia y la Cultura, UNESCO (2018). Plan de Acción 2018-2028. III Conferencia Regional de Educación Superior para América Latina y el Caribe. Instituto Internacional de la UNESCO para la Educación Superior de América Latina y el Caribe (IESALC). https://www.iesalc.unesco.org/2019/02/22/plan-de-accion-cres-2018-2028-2/

Parrino, M. D. C. (2010). Deserción en el primer año universitario. Dificultades y logros. X Coloquio internacional sobre gestión universitaria en América del Sur. Argentina. https://core.ac.uk/download/pdf/30377642.pdf

Qs Latin America Rankings 2019 (2019). QS World University Rankings. Top Universities. https://www.topuniversities.com/university-rankings/latin-american-universityrankings/2019

Ramírez, M. I., \& Montoya, J. (2018). La buena docencia y su evaluación desde el punto de vista de las disciplinas en la Universidad. REDU. Revista de Docencia Universitaria, 16(1), 69-85. https://riunet.upv.es/bitstream/handle/10251/105267/6073-38905-1PB.pdf? sequence $=4$

Ravela, P. (2009). La evaluación del desempeño docente para el desarrollo de las competencias profesionales. En avances y desafíos en la evaluación docente (113-126). Madrid, España:

OEI-Fundación Santillana. https://dialnet.unirioja.es/servlet/articulo?codigo $=4799522$ 
Rodríguez, S., C., Herrera, T., L., Quiles, O., L., \& Álvarez, R., J. (2008). El valor familia en estudiantes universitarios de España: análisis y clasificación. Enseñanza e Investigación en Psicología, 13(2),215-230. https://www.redalyc.org/pdf/292/29213202.pdf

Salgado, L. A. C. (2007). Investigación cualitativa, diseños, evaluación del rigor metodológico y retos. Liberabit, 13(13), 71-80. http://www.scielo.org.pe/scielo.php?script=sci_arttext\&pid=S172948272007000100009

Sayós, R., Pagés, T., Amador, J.A., \& Jorba, H. (2014). Ser buen docente ¿Qué opinan los estudiantes de la universidad de Barcelona?. Revista Iberoamericana de Psicologia y Salud, 5(2), 135-149. https://www.redalyc.org/articulo.oa?id=245131498003

Sgreccia, N., Cirelli, M., \& Vital, M. B. (2015). Cualidades de docentes memorables destacadas por aspirantes a profesor de matemática. Revista de Currículum y Formación del Profesorado, 19(2), 335-350. https://www.redalyc.org/articulo.oa?id=56741181021

Silva L., M. (2011). El primer año universitario: Un tramo crítico para el éxito académico. Perfiles educativos. 33, 102-114. http://www.scielo.org.mx/scielo.php?script=sci_arttext\&pid=S018526982011000500010

Siqueira, V. (2015). Buen profesor universitario desde la perspectiva de los estudiantes. (Tesis de maestría). Universidad ORT Uruguay. https://bibliotecas.ort.edu.uy/bibid/82503/file/2332

Solernou, M., I., A. (2013). La familia y su participación en la universalización de la Educación Superior. Revista Cubana de Educación Médica Superior. 27(1). http://www.ems.sld.cu/index.php/ems/article/view/115

Tapia, C. Amparán, N. Valdez, L. (2017). La enseñanza universitaria eficaz Perspectiva de egresados. En B, Valenzuela. M, Guillén. A, Medina. P, Rodríguez. (Ed.). Educación y Universidad ante el Horizonte 2020 (pp. 213-226). México. http://www.qartuppi.com/2017/HORIZONTE_3.pdf\#page=213

Tapia, C. S., Valdés, A. A., Montes, M. M., \& Valdez, L. A. (2017). Lo que hacen los mejores profesores en una universidad pública mexicana. Revista Praxis Educativa ReDIE, 9(17), 7-25. http://redie.mx/librosyrevistas/revistas/praxiseduc17.pdf

Valdés, M. N. \& Collazo, R. (2013). El buen profesor universitario de Ingeniería y Arquitectura desde la visión de sus estudiantes. Referencia Pedagógica, 1(1) ,78-87. http://rrp.cujae.edu.cu/index.php/rrp/article/view/19/27

Valerio, G., \& Rodríguez, M. C. (2017). Perfil del profesor universitario desde la perspectiva del estudiante. Innovación Educativa, 17(14), $109-124$. https://www.redalyc.org/jatsRepo/1794/179452787007/index.html

Villegas, M.M., \& González, F. (2011). La investigación cualitativa de la vida cotidiana. Medio para la construcción de conocimiento sobre lo social a partir de lo individual. Psicoperspectivas, 10(2), 3559. https://www.redalyc.org/articulo.oa?id=171018843003 
Vries, W. D., León Arenas, P., Romero Muñoz, J. F., \& Hernández Saldaña, I. (2011). ¿Desertores o decepcionados? Distintas causas para abandonar los

estudios universitarios. Revista de la educación superior, 40(160), 29-49. https://www.redalyc.org/articulo.oa?id=60422569002

Zabalza, B. M. A. (2003). Competencias docentes del profesorado, Madrid: Narcea, S.A. De Ediciones.

Zayas, F., Corral, D. I., \& Lugo, D. G. (2011). El involucramiento de padres y madres de familia en la Educación Superior. XI Congreso Nacional de Investigación Educativa. http://www.comie.org.mx/congreso/memoriaelectronica/v11/docs/area_16/0417.pdf 